\title{
Towards a New Democracy: Consensus Through Quantum Parliament
}

\author{
Diederik Aerts \\ Leo Apostel Centre for Interdisciplinary Studies (CLEA) \\ and Foundations of the Exact Sciences (FUND) \\ Department of Mathematics and Department of Psychology \\ Vrije Universiteit Brussel, 1160 Brussels, Belgium \\ E-Mail: diraerts@vub.ac.be
}

\begin{abstract}
We compare different actual forms of democracy and analyse in which way they are variations of a "natural consensus decision process". We analyse how "consensus decision followed by majority voting" is open to "false play" by the majority, and investigate how other types of false play appear in alternative types of democratic decision procedures. We introduce the combined notion of "quantum parliament" and "quantum decision procedure", and prove it to be the only one, when applied after consensus decision, that is immune to false play.
\end{abstract}

\section{Introduction}

The aim of this article is to present a proposal for a new form of democracy. We will give an explicit description of the structure of this new democracy in the sections that follow. I decided to write a contribution on this topic for the book "Worldviews, Science and Us: Redemarcating Knowledge and its Social and Ethical Implications", because I was inspired to elaborate this structure for a new form of democracy primordially while reflecting on the nature of natural processes in the world. It was when reflecting on the nature of "quantum processes" that I had a sudden insight that brought several pieces of a puzzle together. More specifically, it became clear to me how a possible remedy can be proposed for profound shortcomings of the democratic process in our actual society. I found it to be a good example of how reflections on one scientific discipline can lead to fruitful insights in a seemingly completely different scientific field. This proposal for a new form 
of democracy is, however, not a mere attempt to apply insights into the nature of quantum processes to the political processes in our society. It is just as firmly rooted in personal political reflections generated by a long-felt concern about "what is going on with our western democratic systems".

For years I have been formulating, both in private and in circles of close friends, varying critiques of the functioning and practice of our democratic system. I have, however, never made an effort to write down any of these analyses, except for a short text that however remained in the form of a preprint, and its English version 11, 2]. Finally, a variety of reflections came together as pieces of a puzzle, and while I was reflecting on the nature of quantum processes, made me see how a new form of democracy could be presented that would be worthwhile considering as an alternative to our current models.

\section{Democracies}

The word "democracy" originates from the Greek "demos" ("the people") and "kratein" ("to rule"). Hence the original meaning of democracy was: "Rule by the People". When referring to "a democracy", we therefore mean a form of government in which ordinary citizens take part, in contrast with a monarchy or dictatorship [3, 4].

This article concentrates on the type of democracy that is now spreading all over the world, and that emerged in West-Europe in the past centuries, with roots in Ancient Greece [5, 6]. This type of democracy is often called "representative democracy". It comprises a form of government in which voters choose, in free, secret and multi-party elections, representatives to act in their interests. Globally, in 2004, a substantial part of the world's people live in representative democracies, including constitutional monarchies with a strong representative branch [4].

Let us call a representative democracy that works along the mechanism of "majority voting" a "majority rule democracy". In practice, this means that a specific proposal that is debated in a nation's assembly of representatives, e.g. parliament, will be accepted if and only if more than $50 \%$ of such representatives vote in favour. If the $50 \%$ is not attained, the proposal is rejected. A higher percentage of votes in favour is necessary, usually two thirds of the totality of votes, if the proposal introduces a change in the constitution of the nation. This means that the constitution plays the role of a more stable and less easy to change set of rules. 


\subsection{Majority rule democracy}

"The strongest is never strong enough to be always the master, unless he transforms strength into right, and obedience into duty" (Jean Jacques Rousseau in [7])

We want to concentrate on the aspect of majority voting that takes place in a majority rule democracy. Majority voting constitutes in effect a kind of "right of the strongest" in disguise. If in principle the majority always gains, it is the biggest group, and hence the strongest opinion, that always has to be followed after the voting has taken place. One of our critiques of existing democracies is related to this aspect of majority voting. We want to analyse many of its aspects, and hence not only the immoral aspect, as suggested by the above quote from Jean Jacques Rousseau [7. Rousseau argued that "the strongest is never strong enough to be always the master", [7] and hence that the "right of the majority" confers a much more intrinsic power upon the strongest than is the case for the "strongest in nature". Apart from this ethical aspect, we will come to the conclusion that there are other aspects that make majority rule democracy not the best candidate for a democracy.

It is commonly accepted that a majority rule democracy should not be identified as an ethical form of democracy. A majority rule democracy is defended usually for purely pragmatic and practical reasons: it is argued that other types of democracy, the ones that do not adopt the majority rule, are inefficient, because decision making takes too much time and energy of the group of representatives. Let us consider the most important of these other types of democracy, namely the "consensus democracy".

\subsection{Consensus democracy}

Consensus democracy is the application of consensus to the process of legislation. Consensus is a process for group decision-making. It is a method by which an entire group of people can come to an agreement. The input and ideas of all participants are gathered and synthesised to arrive at a final decision acceptable to all.

Consensus decision making is of a higher ethical standard, because it is based on the principle that every voice is worth hearing, and that every concern is justified. If a proposal makes any number of people, even if only one person, deeply unhappy, it is considered that there is a valid reason for that unhappiness, and that ignoring it might be a mistake. The pursuit of consensus not only aims to achieve better solutions but also to foster a sense of community and trust. With consensus, people can and should work 
through differences and reach a mutually satisfactory position. It is possible for one person's insights or strongly held beliefs to sway the whole group. No ideas are lost, each member's input is valued as part of the solution.

But, there are good reasons to be sceptic about a consensus democracy in practice, because indeed it will often take a very long time to reach agreement. This makes a consensus democracy, although ethically of a higher standard than the majority rule democracy, not a very useful form of democracy in practice. It often happens that new institutions start with the implementation of a consensus democratic structure, because members can feel safe then that they will not have to submit to a majority vote decision whose outcome would be very bad for the people they represent. The European Union is an example of an institution that makes use of such a consensus decision structure. Usually, however, such institutions tend to evolve steadily towards a majority rule democracy, the argument being that the consensus democracy "does not work in practice", and leads to too much inefficiency. In the case of the European Union, the complaint is that many important decisions are just not taken, because if one of the members does not agree the result is that "nothing happens". The question: "Why does a consensus democratic system not work in practice?" is one of the key issues that we will try to analyse in this article.

\section{$3 \quad$ Natural and procedural decision processes}

If a group of friends decides to go for a walk in the nearby forest on a sunny Sunday afternoon, their decisions have to be mutually agreed on, e.g. the question "will we stop for a drink in that pub along the way?", will most probably be resolved in some kind of "natural way". What do we mean by "natural"? We mean that no "well defined procedure of how to take decisions" was agreed upon before the friends started out on their walk. This is the way that most of the decisions that involve a group of people are taken in our everyday world. We will call the decision process that takes place in this way a "natural decision process". A decision process that follows a well defined procedure we will call a "procedural decision process".

Sometimes, the distinction between a natural decision process and a procedural decision process is not strict, for example, a process that started as a natural decision process may well end up as a procedural one, if it still fails to yield results after a reasonable amount of time has elapsed. Suppose that during their walk the group of friends gets strongly divided over the question of whether they will have a drink in the pub or not, and that 
they cannot reach agreement including after discussing the matter at some length; it could well be that one of them proposes to vote on the issue, so that the natural decision process is changed into a procedural one, at least, if all agree to this change. As a first remark, we should note that for any type of procedural decision process going on in a group of people it will at least be necessary to have a consensus about the procedure to be followed in the procedural decision process amongst this group of people. However, although this may not seem to be the case at first sight, procedural decision processes have a deeply different structure as compared to natural decision processes. To show this is the subject of the next section.

\subsection{Boycott and false play in procedural decision systems}

Let us look in some more detail at the European Union. For many issues that a majority of the member countries agreed upon, no decisions have been taken, not even in an amended form, because some members, or indeed only a single member nation, did not agree. This has happened on many occasions within the "procedural consensus decision system" that the European Union adopts. We claim that this phenomenon does not typify the "natural decision system". If we return to the group of friends taking a walk on a sunny Sunday afternoon, and if we suppose that no agreement is reached on any of the decisions to be taken, it would be very plausible for the group to decide to have a vote on the matter so as yet to find agreement. But, even before they "decide to have a vote", many other options might have been considered. In any case, what is clear is that most probably the group of friends will not allow their afternoon to be spoiled because a few or only one of them disagrees about what the others want to do, threatening to boycott the entire walk. The reason why such a boycott by a small minority, or one person, rarely happens in everyday life, is because in everyday life no fixed decision procedure has been agreed upon. Friends intending to have a pleasant walk in the forest together will not decide beforehand on a procedure to be followed in case they should stumble upon a disagreement that cannot be resolved without a procedure. The natural decision process is open to any kind of procedure at any moment, and it is exactly for this reason that it cannot be reduced to a specific procedural decision process. A procedural decision process that can be adapted at any moment and as often as required is comparable to the natural decision process, but even such a highly complex procedural decision process would be only an approximation of the natural one. 


\subsection{Pure consensus and majority consensus}

The European Commission follows the simplest consensus decision procedure of all, namely "if no consensus is reached no decision is taken". Let us call this the "pure consensus system". The weakness of this pure consensus system is its vulnerability to boycotting by a small minority, which may even be a single representative. Indeed, if the procedure of the pure consensus system is known by every representative, it will be easy to boycott the whole process by just "not allowing consensus to be reached in the time available".

One of the possibilities to avoid such a boycott is to introduce a different consensus procedure. In this procedure, a consensus is looked for initially, but if it cannot be reached, the procedure of majority voting is followed. Let us call this the "majority consensus system". This procedure is open to false play too, however. If the majority consensus system is adopted, the group of representatives will first look for a consensus, but if no consensus is reached after a given time, which is fixed before the process starts, they will change to the system of majority voting. No boycott of the decision is yet possible, but false play is, obviously. Indeed, the majority may well decide to prevent consensus from being reached, because they know that this will be followed by a majority vote, so that they will have the decision in the way they want it to be, without the need for consensus.

This means that the majority consensus system is open to false play by the majority, just as the pure consensus system is open to boycott by the minority. Both procedures, pure consensus and majority consensus, are very different from the natural decision process that we find around us in everyday life. Can we find a procedural model that resembles more the natural decision process?

\subsection{Random consensus}

Let us make the situation that we are considering somewhat more concrete, such that we can look for alternative procedural decision models. Suppose that an assembly of representatives consisting of $n$ people is gathering, where $n$ is sufficiently large, for example $100 \leq n$. They discuss a specific measure and different decisions in relation with this measure are considered and proposed. Suppose that after a period of discussion two alternative decisions are left, so that the assembly will try to reach consensus considering both of them. However, no consensus ensues in the period of time available. A majority of the representatives, let us say $n-1$, is in favour of decision A, and one representative is in favour of decision $\mathrm{B}$. The two types of decision 
procedures that we have considered so far would produce the following results. The pure consensus system would result in "no decision" being made meaning that, in the perceptions of the $n-1$ persons who are in favour of decision $\mathrm{A}$, the one person in favour of decision $\mathrm{B}$ has boycotted the overall process. The majority consensus system, for its part, would result in decision A being taken.

Suppose that a society using the majority consensus system has become aware of (i) its unethical nature, and also, even more importantly, (ii) the obvious possibility of false play and, as a consequence, of a "forced decision", and suppose that it wants to do something about it. More particularly, a way is investigated to "protect" the minority, in our example, only one person out of $n$, who might, however, be representing a lot of people. The following procedure could be proposed, which we will call the "random consensus system". If no consensus is reached after a well defined period, a random process is organised to determine which of the alternatives will be chosen. In the case of our specific example, this would come to tossing a coin and choosing for decision $\mathrm{A}$ if the coin shows head and decision B if the coin shows tail. Obviously, the minority gains by this random consensus system as compared with the majority consensus system. In the case of our specific example, the one representative gains a lot, because suddenly there is a fiftyfifty chance of decision B or decision A being taken. But if all representatives know in advance that this random consensus system is going to be applied, it can be falsely used by the minority this time, in much the same way as the majority consensus system can be falsely used by the majority. Indeed, the minority, in our case the one representative who is in favour of decision B, may well boycott the process of consensus, because he or she knows that after the time for consensus has passed, the coin will be tossed, leaving him or her with a $50 \%$ chance of his or her preferred decision being taken in its pure form, instead of a consensus decision, which, given that $n-1$ representatives are in favour of decision A, will anyhow be closer to decision $\mathrm{A}$ than to decision B. Our conclusion is that just as the majority consensus system invites false play by the majority, preventing real consensus, the random consensus system invites false play by the minority, equally preventing real consensus.

\section{Quantum democracy}

In the introduction of this article I said that it was reflecting on the nature

of quantum processes that made me see how it would be possible to propose 
a solution to some of the problems of our democratic system. Already years ago, I used to give the example to my students of what I then called a "quantum parliament". Let me explain what such a quantum parliament is, and why I found it an interesting idea at that time.

\subsection{Quantum parliament}

Suppose one considers a classical parliament, such as the ones we know. This means that we have an overall group of representatives constituting the parliament, and subgroups whose members belong to different political parties. Let us be more concrete, and suppose that we have a parliament of $n$ representatives, and that there are five parties, which we will call $\mathrm{A}, \mathrm{B}, \mathrm{C}$, $\mathrm{D}$ and $\mathrm{E}$, where $n_{A}, n_{B}, n_{C}, n_{D}$ and $n_{E}$ are the number of representatives belonging to parties $A, B, C, D$ and $E$. This means that

$$
n_{A}+n_{B}+n_{C}+n_{D}+n_{E}=n
$$

Usually, a government is composed of a collection of parties such that the sum of the representatives of all the composing parties is more than or equal to $n / 2$. As a consequence, whenever the parliament has to vote on a certain proposition, the government can "in some way" obtain a majority vote for this proposition, and hence have it decided the way the government wants. This indeed is the case if all representatives of the parties that constitute the government follow the government's opinion in their parliament vote. We stated "in some way", because in principle this does not have to be and even should not be the case. Indeed, in all western democracies there is a strict division amongst the three powers: (1) the executive power, in the hands of the administrative branch of the government, including ministers, the cabinet, civil servants, the police and the army; (2) the legislative power, in the hands of the lawmakers, effectively the representatives of the parliament; and (3) the judiciary power, more concretely the enforcers of the law, the judges, magistrates and tribunals. But in practice, parliamentary decisions are often made by its members that belong to the government. Apart from this, all parliaments in western democracies decide through majority voting, which means that they give rise to a majority rule democracy.

The quantum parliament follows a probability procedure, hence partly as referred to in section 3.3, but also different. The probabilities are weighted by means of the sizes of the different decision groups. More concretely, this

means the following: we develop a random machinery, such that the parties $A, B, C, D$ and $E$, respectively, are attributed probabilities $p(A)=\frac{n_{A}}{n}$, 
$p(B)=\frac{n_{B}}{n}, p(C)=\frac{n_{C}}{n}, p(D)=\frac{n_{D}}{n}$ and $p(E)=\frac{n_{E}}{n}$. From (11) it follows that

$$
p(A)+p(B)+p(C)+p(D)+p(E)=1
$$

which means that we can interpret $p(A)$ (or $p(B), p(C), p(D), p(E)$, respectively) as the probability of party $A$ (or $B, C, D, E$, respectively) deciding.

Hence the quantum parliament is different from a majority rule parliament, because decisions are taken following a random procedure, which means that also the smallest party can win, but it is also different from a random consensus system, where each party would have an equal chance to win. For each party, the chances to win are proportional to its size; hence the bigger a party, the greater its chance to win.

\subsection{Quantum consensus}

Our proposal for a quantum consensus system is the following. Suppose a specific proposal is made that requires a parliamentary vote. There is a particular period of time available for seeking consensus, which is decided on beforehand. After this time has run out, the quantum parliament is to decide. Concretely, this means that a probabilistic procedure is carried out such that the majority has a probability proportional to its size to win the vote, and equally so the minority has a probability proportional to its size to win the vote. In other words, although the majority has more chance to win, the minority will always have a chance to win too, however small it may be.

It is interesting to point out at this stage that this quantum consensus system is not subject to "false play" and/or boycotting in the way that a pure consensus system, a majority consensus system or a random consensus system is. A pure consensus system can be easily boycotted by the minority, since it will not be followed by a decision vote. The majority consensus system is typically boycotted by the majority. They know that they just have to wait for the voting to win with certainty. The random consensus system is typically boycotted again on the initiative of the minority. Indeed, the minority increases its power by waiting until a pure random decision is made. A quantum consensus system is free from all these flaws. Indeed, if the majority decided to boycott, they might lose the vote to the minority because of the randomness of the procedure. The minority will not be tempted to boycott the consensus either. Although they still have a chance to win if they opt for boycotting the consensus and waiting for the vote, their chance is definitely smaller than that of the majority, which makes this strategy less attractive to them than seeking consensus. 
The quantum consensus system is the only procedure that will stimulate both sides, majority as well as minority, to strive for real consensus. It is the only procedure that avoids that there is any benefit in boycotting the consensus for either side, majority or minority. Nor does it entail the disadvantage of the pure consensus procedure, namely that it takes too long for a decision to follow. In the quantum consensus system, a fixed time is reserved for consensus, after which the quantum parliament can decide in a wink. This means that the quantum consensus system can offer a real, efficient, workable and ethically balanced consensus decision procedure.

\subsection{Natural and procedural, the aspect of determinism}

In this section we reflect about "why there is a fundamental difference between a natural and a procedural decision process", "why the quantum consensus system is a good model for the natural decision process", and "what these insights tell us about the nature of processes in general".

The types of boycotts and false plays that we mentioned in relation with the different versions of procedural decision processes are only possible because decision processes are instruments used by human beings, who have the gift of foresight. Moreover, they are only possible because procedural decision processes contain a definite deterministic aspect. For example, the procedure of a procedural majority decision process is close to deterministic, which means that once the majority and minority are known and fixed, the outcome will be virtually known and fixed. Boycotting and false play find their origin in this possibility of "knowing the future". It is potential future events that influence the present through the minds of the people involved in the process. Human minds manage to create a causal chain from "future potential" to "present actual". Since any procedure that is fixed and free from any randomness increases the potential of the human mind to forecast the future, it also fortifies the causal link between future potential and present actual. Once randomness is introduced in the procedural process, the potential to forecast the future will decrease, approaching the level of a natural decision process. This similarity is most pronounced if the introduced randomness is quantum.

We know that causal effects of potential future to actual present also exist in the realm of the micro-world through the effect of "non-locality". The question arises whether the origin and structure of quantum randomness is not linked to the function we pointed out in relation with decision processes, namely that the quantum consensus system is the only procedural decision process that demotivates the "false play type" of use of this causal "future to 
present effect". There might be a Darwinian element of evolution involved that in the long term makes the quantum process fitter than any other, and such that it was selected in the course of time for the entities populating the micro-world. Our analysis also indicates that the quantum formalism and more specifically the quantum superposition state might deliver a good model for consensus as a state within a process. We plan to investigate these questions in depth within the approach that we put forward in [8, 9, 10, 11.

\section{References}

[1] D. Aerts, Een gebalanceerd stemsysteem voor de democratie, een remedie voor populisme. Preprint Centrum Leo Apostel, Vrije Universiteit Brussel (2000).

[2] D. Aerts, Towards a balanced voting system for democracy, a remedy for populism, Preprint Center Leo Apostel, Vrije Universiteit Brussel (2005).

[3] B. Moore, Social Origins of Dictatorship and Democracy. Lord and Peasant in the Making of the Modern World. Boston: Beacon Press (1966).

[4] A. Lijphart, Democracy in Plural Societies: A Comparative Exploration. New Haven and London: Yale University Press (1977).

[5] M. Levin, The Spectre of Democracy. The Rise of Modern Democracy As Seen By Its Critics. New York: Washington Square Press (1992).

[6] R. A. Dahl, Democracy and Its Critics. New Haven and London: Yale University Press (1989).

[7] J. J. Rousseau, Du Contrat Social ou Pincipes du Droit Politique, Amsterdam: Marc Michel Rey (1762).

[8] D. Aerts, J. Broekaert and L. Gabora, The quantum nature of common processes. Foundations of Science (in press).

[9] D. Aerts, M. Czachor and B. D'Hooghe, Towards a quantum evolutionary scheme: violating Bell's inequalities in language. In: N. Gontier, J. P. Van Bendegem and D. Aerts (Eds.), Evolutionary Epistemology, Language and Culture - a nonadaptationist systems theoretical approach [Theory and Decision Library: Julian Nida-Rümelin]. Dordrecht: Springer (2005). 
[10] D. Aerts and L. Gabora, A theory of concepts and their combinations I\&II. Kybernetes, 34, 151-175, 176-205 (2005).

[11] L. Gabora and D. Aerts, Evolution as context-driven actualization of potential. Interdisciplinary Science Reviews (in press). 